\title{
An Overview of Machining Process of Alumina and Alumina Ceramic Composites
}

\author{
Pravin Pawar*, Raj Ballav, Amaresh Kumar \\ Department of Manufacturing Engineering, National Institute of Technology, Jamshedpur, 831014, India \\ *Corresponding Author: pravin.1900@gmail.com
}

Copyright (C) 2015 Horizon Research Publishing All rights reserved.

\begin{abstract}
The present paper gives a critical review of Alumina and alumina composite ceramic materials machined by different machining process. The article shows that a review on machining of alumina and alumina composites with different input parameters and different output responses such as Material Removal Rate (MRR), Tool Wear Rate ( TWR), Surface Roughness( Ra) etc. using a different mechanism of machining technique, SWOT Analysis \& its summery results of literature review.
\end{abstract}

Keywords Alumina, Material Removal Rate, Tool Wear Rate, Surface Roughness, USM, AWM, EDM, ED Milling, ECDM, SWOT

\section{Introduction}

The machining of advanced nonconductive ceramic like aluminum oxide $\left(\mathrm{Al}_{2} \mathrm{O}_{3}\right)$ ceramic continues to be a major challenge for user industries. Advanced ceramic materials are gradually becoming very important for their superior properties such as high hardness, wear resistance, chemical resistance, low density, superior wear and corrosion resistance, and high-temperature strength and high strength to weight ratio, often result in low material removal rate (MRR), relatively poor surface quality and subsurface damage, which may grow into a spontaneous fracture during machining [3][9][13].

Aluminium oxide $\left(\mathrm{Al}_{2} \mathrm{O}_{3}\right)$ used for making machine tool inserts, aerospace, electrical and electronic components, ballistic armor, ceramic composite automotive brakes, diesel particulate filters, a wide variety of prosthetic products, piezoceramic sensors and next-generation computer memory [2][15]. $\mathrm{Al}_{2} \mathrm{O}_{3}$ is a material with high potential for use in biomedical implants because of its low wear rate and excellent biocompatibility [4][20]

One factor limiting the industrial application of these ceramics is the inability to attain sufficient dimensional control during net-shape fabrication. Thus the additional machining processes is required[14]. Ceramics are known as very difficult to machine materials. The main factors that cause ceramics to be difficult-to-machine are their extreme hardness, non-electrical conductivity and brittleness [11][18][19].

\section{Classification of Machining Process}

\subsection{Ultrasonic Machining Process (USM)}

H.frei et al [1] has experimented on materials of $\mathrm{Al}_{2} \mathrm{O}_{3}$ \& $\mathrm{ZrO}_{2}-\mathrm{Al}_{2} \mathrm{O}_{3}$ using ultrasonic machining process \& after that authors were found out for $\mathrm{Al}_{2} \mathrm{O}_{3}$ strengthening mechanism is depended on a direct correlation between roughness and strength: The lower, roughness the higher the strength. In case of $\mathrm{ZrO}_{2}-\mathrm{Al}_{2} \mathrm{O}_{3}$ there is no direct correlation between strength and roughness. Henrik Dam et al [2] has studied machining process using USM applied into tough and brittle materials and finally results were found out such as production rate, tool wear, and surface roughness. Tai-Chiu Lee et al [7] has studied the effect of whisker orientation on the material removal rate and mechanisms in ultrasonic machining of $\mathrm{SiC}$ whisker-reinforced alumina ceramic composites.

B. Ghahramani et al [8] was taken case study of machining of $\mathrm{Al}_{2} \mathrm{O}_{3}$ using USM and discussed in briefly in USM process, mechanism, dynamics and its trends. Deng Jianxin et al [9] have found out in this research MRR is dependent on the effect of the properties \& Microstructure of the workpiece materials of alumina-based ceramic composites. The experimental results showed that fracture toughness plays a major role with respect to MRR and surface roughness in USM of alumina-based ceramic composites. Z.C. Li et al [14] has produced holes on ceramic matrix composites \& Alumina materials using rotary ultrasonic machining and comparing their results based on cutting force, MRR, chipping thickness and chipping size.

\subsection{Abrasive Waterjet Machining (AWM)}

GI SANG CHOI et al [3] has developed an analytical model for the material removal rate in AWJM and this model was applied into experimentally on Alumina material. The 
results show that material removal rate of AWJM in cutting Brittle and a hard material are high. Also show that abrasion mechanisms for ductile and brittle materials are different.

Manabu Wakuda et al [11] has experimentally identified material removal response of alumina ceramics to the abrasive particles such as aluminum oxide, silicon carbide, synthetic diamond impact in the AJM process. J.wang et al [12] has experimentally analyzed performance of multipass AWJ machining. The results obtained were proved that superior performance using multipass over single passes AWJ cutting on alumina material. D.K. Shanmugam et al [20] has investigated experimentally minimise or eliminate the kerf taper in AWJ cutting of alumina ceramics by using a kerf-taper compensation technique. This technique suggested predicting the kerf taper angle both qualitatively and quantitatively within the tested range of the process parameters.

\subsection{Electro Discharge Machining (EDM)}

J. H. Zhang et al [4] has used electro discharge machine for machining of conductive ceramics $\left(\mathrm{Al}_{2} \mathrm{O}_{3}+\mathrm{TiC}+\mathrm{WC} \mathrm{Hot}\right.$ pressed plate). The output response was discovered out such as material removal rate, surface roughness, and diameter of discharge points by using various input parameters. Biing Hwa Yan et al [6] have to check feasibility of machining of material was $\mathrm{Al}_{2} \mathrm{O}_{3} / 6061 \mathrm{Al}$ using rotary $\mathrm{EDM}$ with ball burnishing. The results were found out machining rate, surface roughness and improvement of surface roughness.

\subsection{Electro Discharge Milling (ED Milling)}

Y.H. Liu et al [18-19] have developed a new novel technique of Electro Discharge milling. In this ED milling author used a thin copper sheet fed to the tool electrode along the surface of the workpiece as the assisting electrode, and uses a water-based emulsion as work fluid to machined insulating $\mathrm{Al}_{2} \mathrm{O}_{3}$ ceramic. This novel technique results shows that high MRR.

Table 1. Literature Review

\begin{tabular}{|c|c|c|c|c|c|c|}
\hline $\begin{array}{l}\text { Ref } \\
\text { No. }\end{array}$ & Mechanism & $\begin{array}{l}\text { W/P Mtl \& Its } \\
\text { Dimensional Shape }\end{array}$ & $\begin{array}{l}\text { Tool Mtl \& Its } \\
\text { Dimensional shape }\end{array}$ & Input Parameters & $\begin{array}{l}\text { Output } \\
\text { Parameters }\end{array}$ & $\begin{array}{l}\text { Reported } \\
\text { By \& Year }\end{array}$ \\
\hline 1 & $\begin{array}{l}\text { Polishing/ } \\
\text { Grinding/ } \\
\text { Ultrasonic } \\
\text { Machining }\end{array}$ & $\begin{array}{l}99.9 \% \mathrm{Al}_{2} \mathrm{O}_{3} \& \\
\mathrm{ZrO}_{2}-\mathrm{Al}_{2} \mathrm{O}_{3}(75.7 \% \\
\mathrm{ZrO}_{2}+ \\
4.2 \% \mathrm{Y}_{2} \mathrm{O}_{3}+20 \% \\
\left.\mathrm{Al}_{2} \mathrm{O}_{3}\right) \text { Rectangular } \\
300 \mu \mathrm{m} \text { thick }\end{array}$ & $\begin{array}{l}\text { Polishing- diamond } \\
\text { particle size of } \\
6-1 \mu \mathrm{m} \text { Grinding- } \\
\text { wheel diamond } \\
\text { particle size of } \\
90 \mu \mathrm{m}\end{array}$ & $\begin{array}{l}\text { Amplitude of tool } \\
20-40 \mu \mathrm{m} \text {, Different } \\
\text { W/P Mtls. }\end{array}$ & $\begin{array}{l}\text { Ra, Strength, } \\
\text { Defects - cracks, } \\
\text { Optimization } \\
\text { Using SEM \& } \\
\text { TEM }\end{array}$ & $\begin{array}{l}\text { H.frei et al } \\
(1992)\end{array}$ \\
\hline 2. & $\begin{array}{l}\text { Ultrasonic } \\
\text { Machining }\end{array}$ & $\mathrm{Al}_{2} \mathrm{O}_{3}$ Ceramic Tiles & $\begin{array}{l}\text { Steel -Tubular Tool } \\
\text { I.D-10mm, } \\
\text { O.D.-8mm }\end{array}$ & $\begin{array}{l}\text { Different W/P } \\
\text { Mtls } \\
\text { Tool rpm-400, } \\
\text { Amplitude-6-50 } \mu \mathrm{m}\end{array}$ & $\begin{array}{l}\text { MRR,TWR, } \\
\text { Ra-9 } \mu \mathrm{m}, \\
\text { Production Rate }\end{array}$ & $\begin{array}{l}\text { Henrik Dam } \\
\text { et al (1995) }\end{array}$ \\
\hline
\end{tabular}

\subsection{Electro Chemical Discharge Machining (ECDM)}

B. Bhattacharya et al [5] has designed and modified ECDM setup and producing novel ECDM setup. In this author developed the modular mechatronic feature of the indigenously designed ECDM set up to machined $\mathrm{Al}_{2} \mathrm{O}_{3}$ ceramic materials. The final output results were measured material removal rate and accuracy of machined materials. Sanjay K. Chak et al [17] has developed unique new technique of trepanning method into ECDM setup to machined $\mathrm{Al}_{2} \mathrm{O}_{3}$ ceramic material. Choosing this technique the author found out the results were improved dimensional accuracy, surface integrity of the machined profile and improvement in the quality of holes produced.

\subsection{Laser Machining}

Chwan-Huei Tsai et al [10] has developed novel designed laser machining applied the concept of fracture machining element. Using this closed cavity for alumina ceramic can be successfully shaped. The results were shows that the material removal rate can be improved when increasing the moving speed of laser.

\subsection{Laser Assisted Machining (LAM)}

Patrick A. Rebro et al [13][15] has used laser-assisted machining (LAM) to machined on $\mathrm{Al}_{2} \mathrm{O}_{3}$ workpieces. Laser-assisted machining is a laser-assisted turning using a Nd:YAG laser. The obtained results applying these techniques give better surface quality, better surface roughness, larger material removal rate and moderate tool wear.

\section{Major Findings on Machining of Alumina \& Alumina Composites}




\begin{tabular}{|c|c|c|c|c|c|c|}
\hline $\begin{array}{l}\text { Ref } \\
\text { No. }\end{array}$ & Mechanism & $\begin{array}{l}\text { W/P Mtl \& Its } \\
\text { Dimensional Shape }\end{array}$ & $\begin{array}{l}\text { Tool Mtl \& Its } \\
\text { Dimensional shape }\end{array}$ & Input Parameters & $\begin{array}{l}\text { Output } \\
\text { Parameters }\end{array}$ & $\begin{array}{l}\text { Reported } \\
\text { By \& Year }\end{array}$ \\
\hline 3 & $\begin{array}{l}\text { Abrasive } \\
\text { Waterjet } \\
\text { Machining }\end{array}$ & $\begin{array}{l}\mathrm{Al}_{2} \mathrm{O}_{3} \mathrm{AD}-94 \\
\text { alumina plates - } \\
5 \mathrm{~mm} \text { thickness. }\end{array}$ & $\begin{array}{l}\text { AISI4340 steel \& } \\
\text { Al2024 aluminium }\end{array}$ & $\begin{array}{l}\text { Abrasive particles - } \\
\varnothing 0.15-0.25 \\
\text { mm, Water Jet } \\
\text { Pressure } \\
\text { (MPa)-150-350, } \\
\text { Types of mtl. }\end{array}$ & $\begin{array}{l}\text { MRR-60- } \\
110 \mathrm{~mm}^{3} / \mathrm{s}, \\
\text { Thikness of } \\
\text { fracture- } 1.5-2.1 \mathrm{~m} \\
\mathrm{~m}, \mathrm{RMS} \text { AE } \\
\text { signal( } 0.1 \mathrm{~V} / \mathrm{div})\end{array}$ & $\begin{array}{l}\text { GI SANG } \\
\text { CHOI et al } \\
(1997)\end{array}$ \\
\hline 4 & $\begin{array}{l}\text { Electro } \\
\text { Discharge } \\
\text { Machining }\end{array}$ & $\begin{array}{l}\mathrm{Al}_{2} \mathrm{O}_{3}+\mathrm{TiC}+\mathrm{WC} \\
\text { hot pressed plate }\end{array}$ & $\begin{array}{l}\text { Copper solid circular } \\
\text { shape }\end{array}$ & $\begin{array}{l}\text { Voltage } 100-180, \\
\text { Current-4-24.8, } \\
\text { Pulse on time-10- } \\
1800 \mu \mathrm{s}, \text { Pulse off } \\
\text { time- } 100-3200 \mu \mathrm{s}\end{array}$ & MRR, TWR,Ra & $\begin{array}{l}\text { J. H. Zhang } \\
\text { et al (1997) }\end{array}$ \\
\hline 5 & $\begin{array}{l}\text { Electro- } \\
\text { Chemical } \\
\text { Discharge } \\
\text { Machining }\end{array}$ & $\begin{array}{l}\mathrm{Al}_{2} \mathrm{O}_{3} \\
25 \mathrm{mmX} 25 \mathrm{mmX} \\
3 \mathrm{~mm}\end{array}$ & Copper of Ø $2 \mathrm{~mm}$ & $\begin{array}{l}\text { electrolyte } \\
\text { concentrations of } \\
20 \%, 25 \% \text { and } 30 \% \\
\text { of } \mathrm{NaOH} \text {, supply } \\
\text { voltage- } 70 \text { to } 90 \mathrm{~V}\end{array}$ & 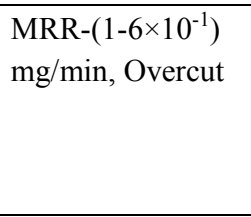 & $\begin{array}{l}\text { B. } \\
\text { Bhattachary } \\
\text { a et al } \\
(1999)\end{array}$ \\
\hline 6 & $\begin{array}{l}\text { Rotary Ball } \\
\text { Burnishing } \\
\text { Electrical } \\
\text { Discharge } \\
\text { Machining }\end{array}$ & $\begin{array}{l}\mathrm{Al}_{2} \mathrm{O}_{3} / 6061 \mathrm{Al} \\
\text { Thickness } 15 \mathrm{~mm} \\
\text { plate }\end{array}$ & $\begin{array}{l}\text { Copper, ring shape } \\
\text { Outer Ø 25.4-25.6 } \\
\text { mm, Inner Ø19 mm }\end{array}$ & $\begin{array}{l}\text { Non-load voltage } \\
\text { (V) } 60-120, \text { Peak } \\
\text { current(Ip)1-15 A, } \\
\text { Pulse duration (ton) } \\
1-650 \mathrm{~ms} \text {, Pulse off } \\
\text { time (toff) 1-650 } \\
\mathrm{ms}\end{array}$ & $\begin{array}{l}\text { MRR-0.15-0.90m } \\
\mathrm{m} / \mathrm{min}, \\
\text { Ra-1.2- } 3.5 \mu \mathrm{m}, \\
\text { ISR-55-92\% }\end{array}$ & $\begin{array}{l}\text { Biing Hwa } \\
\text { Yan et al } \\
(2000)\end{array}$ \\
\hline 7 & $\begin{array}{l}\text { Ultrasonic } \\
\text { Machining }\end{array}$ & $\begin{array}{l}\mathrm{Al}_{2} \mathrm{O}_{3} / \mathrm{SiCw} \\
3 \mathrm{X} 4 \mathrm{X} 36(\mathrm{~mm})\end{array}$ & Mild Steel $\varnothing 5 \mathrm{~mm}$ & $\begin{array}{l}\text { power-250W, } \\
\text { Frequency-16-25kh } \\
\text { z, Amplitude of } \\
\text { vibration-5-20 } \mu \mathrm{m}\end{array}$ & $\begin{array}{l}\text { MRR-0.60-1.65m } \\
\mathrm{m}^{3} / \mathrm{min} \& \\
\mathrm{Ra}-0.4-0.8 \mu \mathrm{m}\end{array}$ & $\begin{array}{l}\text { Tai-Chiu } \\
\text { Lee and } \\
\text { Deng } \\
\text { Jianxin } \\
(2001) \\
\end{array}$ \\
\hline 8 & $\begin{array}{l}\text { Ultrasonic } \\
\text { Machining }\end{array}$ & $\begin{array}{l}\mathrm{Al}_{2} \mathrm{O}_{3} \\
0.18 \times 0.051 \times 0.0095 \\
\mathrm{~m}\end{array}$ & Vibrating Tool & Force $(\mathrm{N}) 72-466$ & $\begin{array}{l}\text { Compressive } \\
\text { Maximum } \\
\left(\mathrm{MN} / \mathrm{m}^{2}\right), \text { Tensile } \\
\text { stress }\left(\mathrm{MN} / \mathrm{m}^{2}\right), \\
\text { Maximum shear } \\
\text { stress }\left(\mathrm{MN} / \mathrm{m}^{2}\right)\end{array}$ & $\begin{array}{l}\text { B. } \\
\text { Ghahramani } \\
\text { et al (2001) }\end{array}$ \\
\hline 9 & $\begin{array}{l}\text { Ultrasonic } \\
\text { Machining }\end{array}$ & $\begin{array}{l}\mathrm{Al}_{2} \mathrm{O}_{3} / \mathrm{TiC}_{2} \mathrm{Al}_{2} \mathrm{O}_{3} / \mathrm{Si} \\
\mathrm{Cw}, \mathrm{Al}_{2} \mathrm{O}_{3} / \mathrm{TiB}_{2}, \\
\text { Size- } 3 \mathrm{X} 4 \mathrm{X} 36(\mathrm{~mm})\end{array}$ & Mild Steel $\varnothing 5$ & $\begin{array}{l}\text { Power-250W, } \\
\text { Frequency-16-25kh } \\
\text { z, Amplitude of } \\
\text { vibration-5-20um }\end{array}$ & $\begin{array}{l}\text { MRR-0.4- } \\
1 \mathrm{~mm}^{3} / \mathrm{min} \\
\mathrm{Ra}-0.5-2 \mu \mathrm{m}\end{array}$ & $\begin{array}{l}\text { Deng } \\
\text { Jianxin et al } \\
(2002)\end{array}$ \\
\hline 10 & $\begin{array}{l}\text { Laser } \\
\text { Machining } \\
\left(\mathrm{CO}_{2} \&\right. \\
\mathrm{Nd}: Y A G)\end{array}$ & $\begin{array}{l}99.6 \% \mathrm{Al}_{2} \mathrm{O}_{3} \\
\text { Substrate } \\
108 \times 108 \times 10(\mathrm{~mm})\end{array}$ & $\begin{array}{l}\text { Wavelength of } \mathrm{CO}_{2} \\
\text { laser }-10.6 \mathrm{~mm}, \mathrm{Dia} \\
\text { of Focus spot }-73 \\
\mathrm{~mm} . \text { wavelength of } \\
\mathrm{Nd} \text { :YAG laser }-1.06 \\
\mathrm{~mm}\end{array}$ & $\begin{array}{l}\text { Power of } \\
\mathrm{Nd} \text { :YAG laser - } 53 \\
\mathrm{~W} \text {, power of } \mathrm{CO}_{2} \\
\text { laser - } 44 \mathrm{~W}\end{array}$ & $\begin{array}{l}\text { MRR- } 0.15 \mathrm{~mm}^{3} / \mathrm{s} \\
\text { Ra- } 60 \mu \mathrm{m}\end{array}$ & $\begin{array}{l}\text { Chwan-Huei } \\
\text { Tsai et al } \\
(2003)\end{array}$ \\
\hline 11 & $\begin{array}{l}\text { Abrasive } \\
\text { Waterjet } \\
\text { Machining }\end{array}$ & $\begin{array}{l}\mathrm{Al}_{2} \mathrm{O}_{3} \\
\text { alumina tiles }\end{array}$ & $\begin{array}{l}\text { Jet pressure (MPa) } \\
-0.3 \text {,Jet distance } \\
(\mathrm{mm})-0.5, \text { Nozzle } \\
(\mathrm{mm})-\varnothing 0.6, \\
\text { Abrasive flow rate } \\
\text { (g/min)-2 Machining } \\
\text { time (s)-20 }\end{array}$ & $\begin{array}{l}\text { Abrasive } \\
\text { Particles-WA, GC, } \\
\text { SD, Abrasive size } \\
(\mathrm{mm})-15-25 \mu \mathrm{m}\end{array}$ & $\begin{array}{l}\text { MRR-(0-0.010), } \\
\text { surface } \\
\text { appearance }\end{array}$ & $\begin{array}{l}\text { Manabu } \\
\text { Wakuda et al } \\
(2003)\end{array}$ \\
\hline
\end{tabular}




\begin{tabular}{|c|c|c|c|c|c|c|}
\hline $\begin{array}{l}\text { Ref } \\
\text { No. }\end{array}$ & Mechanism & $\begin{array}{l}\text { W/P Mtl \& Its } \\
\text { Dimensional Shape }\end{array}$ & $\begin{array}{l}\text { Tool Mtl \& Its } \\
\text { Dimensional shape }\end{array}$ & Input Parameters & $\begin{array}{l}\text { Output } \\
\text { Parameters }\end{array}$ & $\begin{array}{l}\text { Reported } \\
\text { By \& Year }\end{array}$ \\
\hline 12 & $\begin{array}{l}\text { Multipass } \\
\text { abrasive } \\
\text { waterjet } \\
\text { machining }\end{array}$ & $\begin{array}{l}\mathrm{Al}_{2} \mathrm{O}_{3} \\
87 \% \text { alumina } \\
\text { ceramic slabs of } \\
12.7 \mathrm{~mm} \text { thick }\end{array}$ & $\begin{array}{l}\text { Nozzle } \varnothing-1.02 \mathrm{~mm} \\
\text { Orifice } \varnothing-0.39 \mathrm{~mm} \\
\text { Nozzle } \\
\text { Length- } 76.2 \mathrm{~mm} \text {, } \\
\text { Standing } \\
\text { Distance- } 4 \mathrm{~mm} \text {, } \\
\text { Abrasive mass flow } \\
\text { rate: } 8.33 \mathrm{gm} / \mathrm{s}\end{array}$ & $\begin{array}{l}\text { Water } \\
\text { Pressure-345- } \\
\text { 380Mpa Nozzle } \\
\text { transverse } \\
\text { speed(mm),Nozzle } \\
\text { Transverse } \\
\text { Direction-one \& } \\
\text { alternating }\end{array}$ & $\begin{array}{l}\text { Kerf Profile, kerf } \\
\text { taper- } 3.5-8 \\
\text { degree, Surface } \\
\text { roughness- } \\
7-20.4 \mu \mathrm{m}, \text { Depth } \\
\text { of cut-3-12mm }\end{array}$ & $\begin{array}{l}\text { J.wang et al } \\
(2003)\end{array}$ \\
\hline 13 & $\begin{array}{l}\text { Laser } \\
\text { Assisted } \\
\text { Machining }\end{array}$ & $\begin{array}{l}3 \mathrm{Al}_{2} \mathrm{O}_{3}-2 \mathrm{SiO}_{2} \\
10.3 \text { to } 10.6 \mathrm{~mm}\end{array}$ & $\begin{array}{l}1.5 \mathrm{~kW} \mathrm{CO} \text { laser } \\
+60 \mathrm{hpCNC} \text { Turret }\end{array}$ & $\begin{array}{l}\text { Power }(\mathrm{W})-170, \\
\text { Time-(s)-13, Dia D } \\
(\mathrm{mm})-4.30, \\
\text { Length- }(\mathrm{mm})-2.16 \\
\text { Feed- }(\mathrm{mm} / \mathrm{rev}) \\
-0.010 \\
\text { Speed-(rpm) }-800 \\
\text { Dia-d(mm) }-0.50\end{array}$ & $\begin{array}{l}\text { Find out Tool life, } \\
\text { Surface integrity }\end{array}$ & $\begin{array}{l}\text { Patrick A. } \\
\text { Rebro et al } \\
(2004)\end{array}$ \\
\hline 14 & $\begin{array}{l}\text { Rotary } \\
\text { Ultrasonic } \\
\text { Machining }\end{array}$ & $\begin{array}{l}\mathrm{Al}_{2} \mathrm{O}_{3} \\
32 \times 32 \times 6.35(\mathrm{~mm})\end{array}$ & $\begin{array}{l}\text { Diamond core drills } \\
\text { O.D-9.54\& } \\
\text { I.D.-7.82 } \mathrm{mm}\end{array}$ & $\begin{array}{l}\text { Spindle speed } \\
17--50(\mathrm{rev} / \mathrm{s}), \text { Feed } \\
\text { rate } 0.09-.15 \\
(\mathrm{~mm} / \mathrm{s}), \text { Ultrasonic } \\
\text { vibration-Power } \\
\text { supply A: } \\
35 \%-50 \% \text {; }\end{array}$ & $\begin{array}{l}\text { Cutting force, } \\
\text { MRR, chipping } \\
\text { Thickness, and } \\
\text { chipping size }\end{array}$ & $\begin{array}{l}\text { Z.C. Li et al } \\
(2005)\end{array}$ \\
\hline 15 & $\begin{array}{l}\text { Laser } \\
\text { Assisted } \\
\text { Machining }\end{array}$ & $\begin{array}{l}\mathrm{Al}_{2} \mathrm{O}_{3} \\
45 \times 10 \times 10(\mathrm{~mm})\end{array}$ & $\begin{array}{l}25 \mathrm{~W} \text { (continuous } \\
\text { wave) } \mathrm{CO}_{2} \text { laser } \\
\text { TNMG1604 CBN } \\
\text { Tool }\end{array}$ & $\begin{array}{l}\text { Laser power }(\mathrm{W}) \\
25, \text { Laser beam } \\
\text { diameter }(\mathrm{mm}) \\
\text { 1.0,Laser-tool lead } \\
(\mathrm{mm}) 1.0, \text { Depth of } \\
\text { cut }(\mathrm{mm}) 0.05, \\
\text { Feed rate } \\
(\mathrm{m} / \mathrm{min})-0.5-1.0\end{array}$ & $\begin{array}{l}\text { Cutting } \\
\text { force-10-50N, } \\
\operatorname{Ra}(\mu \mathrm{m})-2.98-4.12\end{array}$ & $\begin{array}{l}\text { Chih-Wei } \\
\text { Chang et al } \\
(2007)\end{array}$ \\
\hline 16 & $\begin{array}{l}\text { Laser } \\
\text { Assisted } \\
\text { Machining }\end{array}$ & $\begin{array}{l}\mathrm{Al}_{2} \mathrm{O}_{3} \\
\varnothing 15 \mathrm{~mm} \& 65 \mathrm{~mm} \\
\text { length }\end{array}$ & $\begin{array}{l}\text { Beam spot diameter } \\
\text { of } 0.54 \mathrm{~mm} \text {, A focal } \\
\text { length of } 40 \mathrm{~mm} \text {, } \\
\text { CBN Tool }\end{array}$ & $\begin{array}{l}\text { Depth of cut }(\mathrm{mm}) \\
-0.2-1 \text { Rotational } \\
\text { speed } \\
(\mathrm{rpm})-1000-2000, \mathrm{~F} \\
\text { eed } \\
(\mathrm{mm} / \mathrm{rev})-0.01-0.03 \\
, \text { Pulsed frequency } \\
(\mathrm{kHz})-30-50\end{array}$ & $\begin{array}{l}\mathrm{Ra}(\mu \mathrm{m})-0.24- \\
1.18 \\
\operatorname{MRR}\left(\mathrm{mm}^{3} / \mathrm{s}\right) \\
-1.5708-23.5619\end{array}$ & $\begin{array}{l}\text { Chih-Wei } \\
\text { Chang et al } \\
(2007)\end{array}$ \\
\hline 17 & $\begin{array}{l}\text { Electro-Chem } \\
\text { ical } \\
\text { Discharge } \\
\text { Machining }\end{array}$ & $\begin{array}{l}\mathrm{Al}_{2} \mathrm{O}_{3} \\
15 \times 15 \times 7 \mathrm{~mm}^{3}\end{array}$ & $\begin{array}{l}\text { A spring fed } \\
\text { diamond embedded } \\
\text { cylindrical abrasive } \\
\text { tool electrode of } \\
\varnothing 1.5 \mathrm{~mm}\end{array}$ & $\begin{array}{l}\text { Voltage, X1 } \\
\text { (V)-60-120,Duty } \\
\text { factor, X2 } \\
(\%)-0.48-0.96 \\
\text { Electrolyte } \\
\text { conductivity, } \\
\text { X3 (mmho/cm) - } \\
275-375\end{array}$ & $\begin{array}{l}\text { Dimensional } \\
\text { accuracy and } \\
\text { surface integrity }\end{array}$ & $\begin{array}{l}\text { Sanjay K. } \\
\text { Chak et al } \\
(2007)\end{array}$ \\
\hline 18 & $\begin{array}{l}\text { Electrical } \\
\text { Discharge } \\
\text { Millling }\end{array}$ & $\begin{array}{l}\mathrm{Al}_{2} \mathrm{O}_{3} \\
\text { Rectangular shape }\end{array}$ & $\begin{array}{l}\text { Steel, } \\
\text { Assisting electrode } \\
\text { material red copper }\end{array}$ & $\begin{array}{l}\text { Pulse time } 500 \mathrm{~ms} \\
\text { pulse off-time- } 400 \\
\text { ms, peak } \\
\text { current-25A } \\
\text { Positive polarity, } \\
\text { Machining fluid } \\
\text { water-based }\end{array}$ & $\begin{array}{l}\operatorname{MRR}(\mathrm{mg} / \mathrm{min})-10 \\
0-300 \\
\operatorname{Ra}(\mu \mathrm{m})-6-10\end{array}$ & $\begin{array}{l}\text { Y.H. Liu et } \\
\text { al (2008) }\end{array}$ \\
\hline
\end{tabular}




\begin{tabular}{|c|c|c|c|c|c|c|}
\hline $\begin{array}{l}\text { Ref } \\
\text { No. }\end{array}$ & Mechanism & $\begin{array}{l}\text { W/P Mtl \& Its } \\
\text { Dimensional Shape }\end{array}$ & $\begin{array}{l}\text { Tool Mtl \& Its } \\
\text { Dimensional shape }\end{array}$ & Input Parameters & $\begin{array}{l}\text { Output } \\
\text { Parameters }\end{array}$ & $\begin{array}{l}\text { Reported } \\
\text { By \& Year }\end{array}$ \\
\hline 19 & $\begin{array}{l}\text { Electrical } \\
\text { Discharge } \\
\text { Millling }\end{array}$ & $\begin{array}{ll}\mathrm{Al}_{2} \mathrm{O}_{3} & \text { Rectangle } \\
\text { shape } & \end{array}$ & $\begin{array}{l}\text { Steel \& Assisted } \\
\text { electrode Mtl red } \\
\text { copper, shape- } \\
\text { Circular wheel }\end{array}$ & $\begin{array}{l}\text { Pulse duration- } \\
50-500 \mu \mathrm{s}, \text { Peak } \\
\text { Voltage- } 100-200, \\
\text { Peak Current- } 25 \mathrm{~A} \text {, } \\
\text { Feed speed } \\
\text { (mm/min)-0.5-3, } \\
\text { Rotational } \\
\text { Speed(r/min)-250-1 } \\
500\end{array}$ & $\begin{array}{l}\text { MRR } 120-350 \\
\mathrm{mg} / \mathrm{min}, \\
\mathrm{Ra}-8-12 \mu \mathrm{m}\end{array}$ & $\begin{array}{l}\text { Y.H. Liu et } \\
\text { al (2008) }\end{array}$ \\
\hline 20 & $\begin{array}{l}\text { Abrasive } \\
\text { Waterjet } \\
\text { Machining }\end{array}$ & $\begin{array}{l}\mathrm{Al}_{2} \mathrm{O}_{3} \\
\text { ceramic tiles } \\
150 \times 100 \times 12.7 \mathrm{~mm}\end{array}$ & $\begin{array}{l}\text { 76.2-mm-long } \\
\text { carbide nozzle } \\
\text { (or mixing tube) of } \\
0.762 \mathrm{~mm} \text { in Dia }\end{array}$ & $\begin{array}{l}\text { Traverse speed } \\
(\mathrm{mm} / \mathrm{s})-0.67-1.17, \\
\text { Standoff distance } \\
(\mathrm{mm})-2-5, \text { Water } \\
\text { pressure } \\
(\mathrm{MPa})-310-410 \text {, } \\
\text { Abrasive mass flow } \\
\text { rate (g/s)-7.6- 9, } \\
\text { Compensation } \\
\text { angle(degree)-0-5 }\end{array}$ & $\begin{array}{l}\text { kerf taper } \\
\text { Angle- }\left(1-5^{\circ}\right)\end{array}$ & $\begin{array}{l}\text { D.K. } \\
\text { Shanmugam } \\
\text { et al (2008) }\end{array}$ \\
\hline
\end{tabular}

\section{SWOT Analysis on Machining Process of Alumina \& Alumina Composites}

Based on the literature survey of machining process of alumina \& alumina composites the following points were drawn form of discussion on analysis Strength, Weakness, Opportunities \& Threats (SWOT).

\subsection{Strengths}

Advanced ceramic material have superior properties such as high hardness and strength at elevated temperatures, chemical inertness, high wear resistance, low thermal conductivity, high strength to weight ratio, high corrosion resistance, oxidation resistance, lower thermal expansion coefficient, low density, high-temperature stability, light weight, high compressive strength, a stronger electromagnetic response than that of metals and good creep resistance due to this it is used as wide application.

\subsection{Weakness}

1. Ceramics are known as very difficult-to-machine materials. The main factors that cause ceramics to be difficult to machine are their high hardness, non-electrical conductivity and brittleness.

2. Ceramic components often to meet high demands for dimensional accuracy and surface quality.

3. Due to different material removing processes the various machining methods lead to different type material damage.
4. The tougher the material, the greater is the tool wear \& lesser is the machining rates.

5. The ceramic material is more brittle in nature due to this the greater is the tendency for removal of debris by fracture.

\subsection{Opportunities}

1. Mechanical industries ceramic uses such in making seals, valves, aerospace, ballistic armor, ceramic composite automotive brakes, diesel particulate filters, a wide variety of prosthetic products, bearings, water pump seals, the adiabatic material of catalytic converters, roller followers, rotors, and cutting tools.

2. Electronic application such as substrate in hybrid circuits, piezo-ceramic sensors and next generation computer-memory.

3. Biomedical is making components such as artificial joints, bones, and teeth.

\subsection{Threats}

The characteristics of machining-induced damage depend also on the properties of the machined ceramic due to this reason it is impossible for machined ceramic material by using conventional machining process for that purpose only non-conventional process is only beneficial.

1. Conventional machining of ceramic is not only difficult but also costly attempt

2. Most of the ceramic parts shaped by sintering processes cannot meet the requirements of accuracy and surface quality. Therefore the machining and surface finishing of parts become necessary 


\section{Results \& Observations}

The Bar Chart Shows that numbers of papers published with respect to their Machining process. The Pie Chart shows that percentage of research conducted in different machining process on alumina ceramic materials.

\section{Number of Published work}

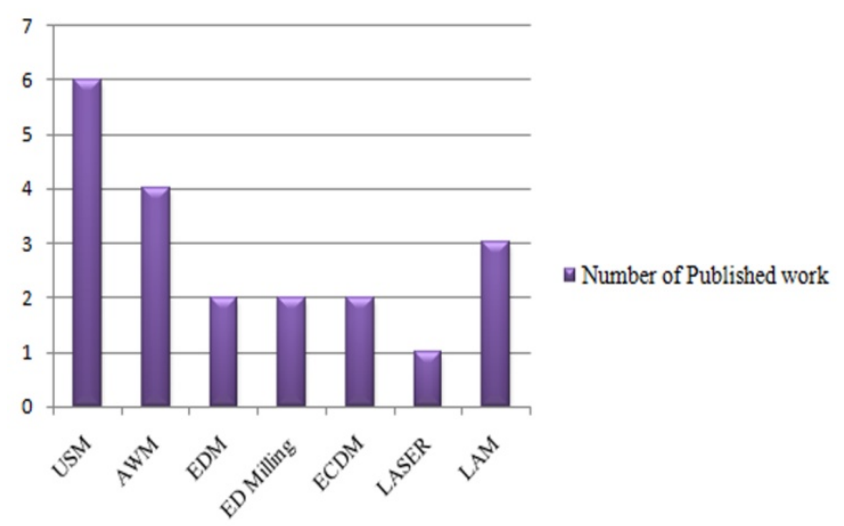

Figure 1. Distribution of the collected research papers for machining on Alumina ceramic materials
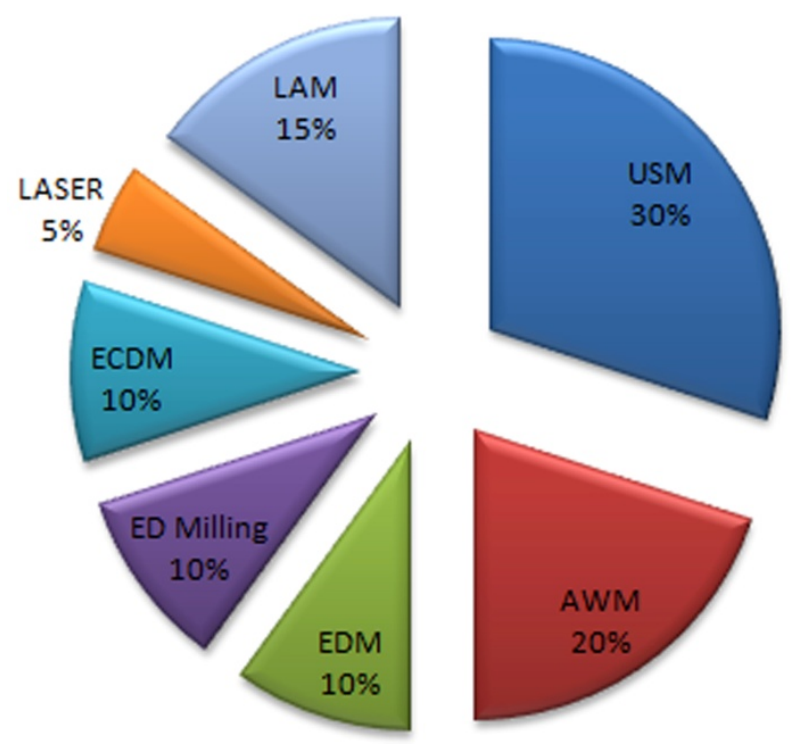

Figure 2. Percentage of Research conducted in different machining process on Alumina ceramic materials

\section{Conclusion}

1. The given literature review table 1 shows that machining of $\mathrm{Al}_{2} \mathrm{O}_{3} \& \mathrm{Al}_{2} \mathrm{O}_{3}$ composites by different machining process mechanism such that uses $30 \%$ of Ultrasonic Machining process, 20\% of Abrasive Water-jet Machining process, $15 \%$ of Laser Assisted Machining process, $10 \%$ of Electro Discharge Machining process, $10 \%$ of Electro Discharge Milling \& 10\% of Electro Chemical Discharge Machining Process \& 5\% of Laser Machining.

2. Maximum authors put efforts to find output responses such as MRR, TWR, Ra \& some of them find out Cutting force, Dimensional accuracy, Analysis of Depth of cut, Tool life, surface integrity \& also comparing different material results.

3. The review reveals that different authors use different machining process by taking different work-piece \& tool materials $\&$ also taking different input $\&$ output parameters to find out optimum results.

\section{Acknowledgements}

This paper is a revised and expanded version of a paper entitled 'A Review on machining process of alumina and alumina composite ceramics' presented at International Conference on Industrial, Mechanical and Production Engineering: Advancements and Current Trends (ICIMPACT) held during 27th to 29th November 2014 in MANIT, Bhopal, India.

\section{REFERENCES}

[1] H Frei., G Grathwohl. Microstructure and strength of Advanced Ceramics after machining. ceramics International ,19,93-104, 1993

[2] Henrik Dam, Per Quist, Mads Peter Schreiber. Productivity surface quality \& tolerances in ultrasonic machining of ceramics, Journal of Material Processing Technology, $51,358-368,1995$

[3] Gi Sang Choi, Gi Heung Choi. Process analysis and monitoring in abrasive water jet machining of alumina ceramics. International Journal of Machine Tools \& Manufacture, Vol -37, no.3, 295-307, 1997

[4] J. H. Zhang., T. C. Lee band W. S. Lau Study on the electro-discharge machining of a hot pressed aluminum oxide based ceramic. Journal of Materials Processing Technology, 63, 908-912, 1997

[5] B. Bhattacharyya, B.N. Doloi, S.K. Sorkhel. Experimental investigations into electrochemical discharge machining (ECDM) of non-conductive ceramic materials. Journal of Materials Processing Technology, 95, (145-154), 1999

[6] Biing Hwa Yan, Che Chung Wang, Han Ming Chow, Yan Cherng Lin. Feasibility study of rotary electrical discharge machining with ball burnishing for $\mathrm{Al}_{2} \mathrm{O}_{3} / 6061 \mathrm{Al}$ composite. International Journal of Machine Tools \& Manufacture, 40, 1403-1421, 2000

[7] Tai-Chiu Lee, Deng Jianxin. Ultrasonic erosion of whisker reinforced ceramic composites. Ceramics International, 27, 755-760, 2001

[8] B. Ghahramani, Z.Y. Wang. Precision ultrasonic machining process a case study of stress analysis of ceramic $\left(\mathrm{Al}_{2} \mathrm{O}_{3}\right)$. International Journal of Machine Tools \& Manufacture, 41, $1189-1208,2001$

[9] Deng Jianxin, Lee Taichiu. Ultrasonic machining of alumina based ceramic composites, Journal of the European Ceramic 
Society, 22,1235-1241,2002

[10] Chwan Huei Tsai, Hong Wen Chen. Laser milling of cavity in ceramic substrate by fracture-machining element technique. Journal of Materials Processing Technology, 136, 158-165 , 2003

[11] Manabu Wakuda, Yukihiko Yamauchi, Shuzo Kanzaki. The cutting performance in multipass abrasive waterjet machining of industrial ceramics, Journal of Materials Processing Technology, 132 ,177-183,2003

[12] J.wang , D.M. Guo. The cutting performance in multipass abrasive waterjet machining of industrial ceramics Journal of Materials Processing Technology, 133,371-377, 2003

[13] Patrick A. Rebro, Yung C. Shin, Frank P. Incropera Design of operating conditions for crackfree laser-assisted machining of mullite International Journal of Machine Tools \& Manufacture 44, 677-694,2004

[14] Z.C. Li, Y. Jiao, T.W. Deines, Z.J. Pei, C. Treadwell Rotary ultrasonic machining of ceramic matrix composites feasibility study and designed experiments. International Journal of Machine Tools \& Manufacture, 45, 1402-1411,2005

[15] Chih-Wei Chang, Chun-Pao Kuo Evaluation of surface roughness in laser-assisted machining of aluminum oxide ceramics with Taguchi method. International Journal of Machine Tools \& Manufacture, 47,141-147,2007

[16] Chih-Wei Chang, Chun-Pao Kuo. An investigation of laser-assisted machining of $\mathrm{Al}_{2} \mathrm{O}_{3}$ ceramics planning. International Journal of Machine Tools \& Manufacture,47,452-461,2007

[17] Sanjay K. Chak, P. Venkateswara Rao. Trepanning of $\mathrm{Al}_{2} \mathrm{O}_{3}$ by electro-chemical discharge machining (ECDM) process using abrasive electrode with pulsed DC supply. International Journal of Machine Tools \& Manufacture,47,2061-2070,2007

[18] Y.H. Liu, X.P. Li, R.J. Ji, L.L. Yu, H.F. Zhang, Q.Y. Li. Effect of technological parameter on the process performance for electric discharge milling of insulating $\mathrm{Al}_{2} \mathrm{O}_{3}$ ceramic. Journal of Matl. Processing Technology , 208 , 245-250,2008

[19] Y.H. Liu, R.J. Ji, X.P. Li, L.L. Yu, H.F. Zhang, Q.Y. Li. Effect of machining fluid on the process performance of electric discharge milling of insulating $\mathrm{Al}_{2} \mathrm{O}_{3}$ ceramic. International Journal of Machine Tools \& Manufacture,48, 1030-1035,2008

[20] D.K. Shanmugam, J. Wang, H. Liu. Minimisation of kerf tapers in abrasive waterjet machining of alumina ceramics using a compensation technique. International Journal of Machine Tools \& Manufacture,48,1527-1534,2008 